\title{
Accelerated Series for Riemann Zeta Function at Odd Integer Arguments
}

\author{
Juuso T. Olkkonen ${ }^{1}$, Hannu Olkkonen ${ }^{2}$ \\ ${ }^{1}$ VTT Technical Research Centre of Finland, Espoo, Finland \\ ${ }^{2}$ Department of Applied Physics, University of Eastern Finland, Kuopio, Finland \\ Email: juuso.olkkonen@vtt.fi, hannu.olkkonen@uef.fi
}

Received August 29, 2012; revised September 29, 2012; accepted October 12, 2012

\begin{abstract}
Riemann zeta function is an important tool in signal analysis and number theory. Applications of the zeta function include e.g. the generation of irrational and prime numbers. In this work we present a new accelerated series for Riemann zeta function. As an application we describe the recursive algorithm for computation of the zeta function at odd integer arguments.
\end{abstract}

Keywords: Riemann Zeta Function; Converging Series; Number Theory; Cryptography; Signal Processing; Compressive Sensing

\section{Introduction}

The Riemann zeta function $\zeta(s)$ defined as for complex numbers s with $R\{s\}>1$

$$
\zeta(s)=\sum_{n=1}^{\infty} \frac{1}{n^{s}}=\prod_{p \text { prime }}\left(1-\frac{1}{p^{s}}\right)^{-1}
$$

has a central role in number theory and appears in many areas of science and technology [1]. The Riemann zeta function is closely related to the prime numbers via (1) and it is an important tool in cryptography. Algorithms for evaluating $\zeta(s)$ are vitally developing initialised by Euler, who invented the basic equation (1). Much of the research effort has been laid to the developing the accelerated series for computation of $\zeta(s)$ for integer arguments [1-4]. For even $s$, the Riemann zeta function obeys the general rule $\zeta(s)=\pi^{s} / N(s)$, where $N(s)$ is an integer. For odd s, no closed form solution for $\zeta(s)$ has been solved and the computational algorithms are usually based on the acceleration of the series (1) by asymptotic expansion with Bernoulli numbers or via Euler's transformation [2]. Recursive algorithms have also presented for evaluation of $\zeta(s)$ at odd integers [5].

In this work we describe a new accelerated series for the Riemann zeta function at integer arguments. The main result is involved in Theorem 1.

Theorem 1: Let us suppose that $\zeta(s)$ is the Riemann zeta function defined by (1). The following series converges as

$$
\sum_{s=0}^{\infty} \frac{\zeta(s+2)}{s !}=\zeta(2)+\sum_{n=1}^{\infty} \frac{\mathrm{e}^{1 / n}-1}{n^{2}}
$$

In Section 2 we give the proof of Theorem 1. In Section 3 we present derivatives of Theorem 1 and describe the method for accelerating the zeta function series given by Theorem 1. In Section 4 we describe the recursive algorithm for evaluation of the Riemann zeta function at integer arguments.

\section{Proof of Theorem 1}

We may deduce

$$
\begin{aligned}
\sum_{s=0}^{\infty} \frac{\zeta(s+2)}{s !} & =\sum_{s=0}^{\infty} \frac{1}{s !} \sum_{n=1}^{\infty} \frac{1}{n^{s+2}} \\
& =\sum_{n=1}^{\infty} \frac{1}{n^{2}} \sum_{s=0}^{\infty} \frac{n^{-s}}{s !}=\sum_{n=1}^{\infty} \frac{1}{n^{2}} \mathrm{e}^{1 / n}
\end{aligned}
$$

The series (2) converges very slowly. However, we may write

$$
\sum_{n=1}^{\infty} \frac{1}{n^{2}} \mathrm{e}^{1 / n}=\sum_{n=1}^{\infty} \frac{\mathrm{e}^{1 / n}-1+1}{n^{2}}=\zeta(2)+\sum_{n=1}^{\infty} \frac{\mathrm{e}^{1 / n}-1}{n^{2}}
$$

which has an accelerated convergence. The proof is now completed.

\section{Derivatives of Theorem 1}

Lemma 1: For $m \geq 2$

$$
\sum_{s=0}^{\infty} \frac{\zeta(s+m)}{s !}=\zeta(m)+\sum_{n=1}^{\infty} \frac{\mathrm{e}^{1 / n}-1}{n^{m}}
$$


Proof: Similar as Theorem 1 .

Lemma 2: For $m \geq 2$

$$
\sum_{s=1}^{\infty} \frac{\zeta(s+m)}{s !}=\sum_{n=1}^{\infty} \frac{\mathrm{e}^{1 / n}-1}{n^{m}}
$$

Proof: Follows directly from Lemma 1 by elimination of the first term in series (4).

Lemma 3:

$$
\sum_{s=0}^{\infty} \frac{\zeta(2 s+2)}{s !}=\zeta(2)+\sum_{n=1}^{\infty} \frac{\mathrm{e}^{1 / n^{2}}-1}{n^{2}}
$$

Proof: Similar as Theorem 1.

Lemma 4:

$$
\sum_{s=0}^{\infty} \frac{\zeta(2 s+3)}{s !}=\zeta(3)+\sum_{n=1}^{\infty} \frac{\mathrm{e}^{1 / n^{2}}-1}{n^{3}}
$$

Proof: Follows directly Lemma 3.

Lemmas 3 and 4 can be generalised as

Lemma 5: For $m \geq 2$

$$
\sum_{s=0}^{\infty} \frac{\zeta(2 s+m)}{s !}=\zeta(m)+\sum_{n=1}^{\infty} \frac{\mathrm{e}^{1 / n^{2}}-1}{n^{m}}
$$

Lemma 6: For $m \geq 2$

$$
\sum_{s=1}^{\infty} \frac{\zeta(2 s+m)}{s !}=\sum_{n=1}^{\infty} \frac{\mathrm{e}^{1 / n^{2}}-1}{n^{m}}
$$

The last series (Lemma 6) can be further generalized as

Lemma 7: For $k \geq 1$ and $m \geq 2$

$$
\sum_{s=1}^{\infty} \frac{\zeta(k s+m)}{s !}=\sum_{n=1}^{\infty} \frac{\mathrm{e}^{1 / n^{k}}-1}{n^{m}}
$$

The series can be further accelerated by noting that $\lim _{s \rightarrow \infty} \zeta(s)=1$. We may write Lemma 7 as

$$
\begin{aligned}
& \sum_{s=1}^{\infty} \frac{\zeta(k s+m)-1+1}{s !} \\
& =\sum_{s=1}^{\infty} \frac{\zeta(k s+m)-1}{s !}+\sum_{s=1}^{\infty} \frac{1}{s !}=\sum_{n=1}^{\infty} \frac{\mathrm{e}^{1 / n^{k}}-1}{n^{m}}
\end{aligned}
$$

which gives

Lemma 8: For $k \geq 1$ and $m \geq 2$

$$
\sum_{s=1}^{\infty} \frac{\zeta(k s+m)-1}{s !}=1-\mathrm{e}+\sum_{n=1}^{\infty} \frac{\mathrm{e}^{1 / n^{k}}-1}{n^{m}} .
$$

\section{Recursive Algorithm}

From Lemma 8 we may deduce

Lemma 9: For $k \geq 1$ and $m \geq 2$

$$
\zeta(k+m)=2-\mathrm{e}+\sum_{n=1}^{\infty} \frac{\mathrm{e}^{1 / n^{k}}-1}{n^{m}}-\sum_{s=2}^{\infty} \frac{\zeta(k s+m)-1}{s !}
$$

The last series can be computed if $\zeta(k s+m)$, $s=2,3, \cdots$ are known. This leads to the fast recursive computation of the zeta function. Especially for $k=1$ we obtain the sequential $\zeta(m+1)$ values as

$$
\zeta(m+1)=2-\mathrm{e}+\sum_{n=1}^{\infty} \frac{\mathrm{e}^{1 / n}-1}{n^{m}}-\sum_{s=2}^{\infty} \frac{\zeta(m+s)-1}{s !}
$$

Both series in (13) have accelerated convergence and to obtain the required accuracy only a few previously computed $\zeta(m+s), s=2,3, \cdots$ values are needed.

\section{Discussion}

In this work we present a new accelerated series for Riemann zeta function. The key observation is presented in Theorem 1. The infinite summation of the zeta functions weighted by $1 / s$ ! can be represented by fast converging series. One application is the recursive computation of the zeta function from the sequence of previously known zeta function values. The recursive algorithm can be initialised using (13), which has itself accelerated convergence. Especially in high values of $m$ the first series in (13) has high convergence due to the term $n^{m}$ in the denominator.

The zeta function values for odd integers are generally believed to be irrational, thought consistent proof is given only for $\zeta(3)$ [6,7]. The irrational number sequences, which can be easily reproduced from a few parameters are important e.g. in encryption coding. The recursive algorithm (Lemma 9) serves as a good candidate for the irrational number generator, since it requires only two parameters $(k$ and $m)$. By altering the parameters a countless number of irrational number sequences are obtained.

Recently a close connection with the log-time sampled signals and the zeta function has been observed [8]. The zeta transform allows the analysis and synthesis of the log-time sampled signals for example in compressive sensing applications.

\section{Acknowledgements}

This work was supported by the National Technology Agency of Finland (TEKES). The authors would like to thank the anonymous reviewers for their valuable comments.

\section{REFERENCES}

[1] J. M. Borwein, D. M. Bradley and R. E. Crandall, "Computational Strategies for the Riemann Zeta Function," Journal of Computational and Applied Mathematics, Vol. 121, No. 1-2, 2000, pp. 247-296. doi:10.1016/S0377-0427(00)00336-8

[2] E. Grosswald, "Remarks Concerning the Values of the 
Riemann Zeta Function at Integral, Odd Arguments," Journal of Number Theory, Vol. 4, No. 3, 1972, pp. 225235. doi:10.1016/0022-314X(72)90049-2

[3] D. Cvijović and J. Klinowski, "Integral Representations of the Riemann Zeta Function for Odd-Integer Arguments," Journal of Computational and Applied Mathematics, Vol. 142, No. 2, 2002, pp. 435-439. doi:10.1016/S0377-0427(02)00358-8

[4] T. Ito, "On an Integral Representation of Special Values of the Zeta Function at Odd Integers," Journal of Mathematical Society of Japan, Vol. 58, No. 3, 2006, pp. 681691. doi:10.2969/jmsj/1156342033

[5] H. Olkkonen and J. T. Olkkonen, "Fast Converging Se- ries for Riemann Zeta Function," Open Journal of Discrete Mathematics, Vol. 2, No. 4, 2012, pp. 131-133. doi:10.4236/ojdm.2012.24025

[6] R. Apéry, "Irrationalité de $\zeta(2)$ et $\zeta(3)$," Astérisque, Vol. 61, 1979, pp. 11-13.

[7] F. Beukers, "A Note on the Irrationality of $\zeta(2)$ and $\zeta(3)$," Bulletin London Mathematical Society, Vol. 11, No. 3, 1979, pp. 268-272. doi:10.1112/blms/11.3.268

[8] H. Olkkonen and J. T. Olkkonen, "Log-Time Sampling of Signals: Zeta Transform," Open Journal of Discrete Mathematics, Vol. 1, No. 2, 2011, pp. 62-65. doi:10.4236/ojdm.2011.12008 